\title{
Differential Effects of Nonselective Versus Selective $\beta$-Blockers on Cardiac Sympathetic Activity and Hemostasis in Patients with Heart Failure
}

\author{
Olav R. de Peuter ${ }^{1}$, Hein J. Verberne ${ }^{2}$, Wouter E. Kok $^{3}$, Bas van den Bogaard ${ }^{1}$, Marianna C. Schaap ${ }^{4}$, Rienk Nieuwland ${ }^{4}$, \\ Joost C.M. Meijers ${ }^{1,5}$, G. Aernout Somsen ${ }^{6}$, Ad Bakx ${ }^{7}$, and Pieter W. Kamphuisen ${ }^{1,8}$ \\ ${ }^{1}$ Department of Vascular Medicine, Academic Medical Center, University of Amsterdam, Amsterdam, The Netherlands; ${ }^{2}$ Department \\ of Nuclear Medicine, Academic Medical Center, University of Amsterdam, Amsterdam, The Netherlands; ${ }^{3}$ Department of Cardiology, \\ Academic Medical Center, University of Amsterdam, Amsterdam, The Netherlands; ${ }^{4}$ Department of Experimental Clinical Chemistry, \\ Academic Medical Center, University of Amsterdam, Amsterdam, The Netherlands; ${ }^{5}$ Department of Experimental Vascular Medicine, \\ Academic Medical Center, University of Amsterdam, Amsterdam, The Netherlands; ${ }^{6}$ Department of Cardiology, Onze Lieve Vrouwe \\ Gasthuis, Amsterdam, The Netherlands; ${ }^{7}$ Department of Cardiology, BovenIJ Hospital, Amsterdam, The Netherlands; and \\ ${ }^{8}$ Department of Vascular Medicine, University Medical Center Groningen, University of Groningen, Groningen, The Netherlands
}

Carvedilol, a nonselective $\beta$-blocker, may be more effective than the selective $\beta$-blocker metoprolol in reducing the risk of thromboembolic events in heart failure. The aim of this study was, first, to assess whether there is a differential response in cardiac sympathetic activity by ${ }^{123}$ I-meta-iodobenzylguanidine (123/-MIBG) imaging when either $\beta$-blocker is used. Second, we assessed whether that response correlates with levels of various serum factors that serve as markers for coagulability. Methods: In this prospective, randomized, open-label crossover study with masked outcome assessments, stable heart failure patients (left ventricular ejection fraction $<40 \%$ ) homozygous for the $\operatorname{Arg} 16 / \mathrm{G} \ln 27(n=13)$ or Gly16/Glu27 haplotype $(n=8)$ of the $\beta_{2}$-receptor were randomized to equipotent dosages of carvedilol or metoprolol for two 6-wk periods. Primary outcome was sympathetic activity as measured by ${ }^{123}$ |-MIBG myocardial washout. Secondary outcomes included markers of hemostasis. Results: ${ }^{123}$ I-MIBG cardiac washout was lower during carvedilol than metoprolol treatment $(12.9 \% \pm 3.9 \%$ vs. $22.1 \% \pm 2.8 \%$, respectively, $P=0.003$ ), irrespective of $\beta_{2}$-adrenergic receptor haplotype. In addition, treatment with carvedilol resulted in a lower von Willebrand factor than did metoprolol $(149 \% \pm 13 \%$ vs. $157 \% \pm$ $13 \%$, respectively, $P=0.01$ ), irrespective of $\beta_{2}$-adrenergic receptor haplotype. Conclusion: Compared with metoprolol, carvedilol resulted in greater reduction of sympathetic activity after 6 wk of treatment and lower von Willebrand factor concentrations in both Arg16/ Gln27 and Gly16/Glu27 individuals. Therefore, carvedilol may reduce the risk of thromboembolic events in patients with heart failure, irrespective of $\beta_{2}$-receptor haplotype status.

Key Words: heart failure; $\beta$-blockers; genetics, coagulation; sympathetic nervous system

J Nucl Med 2013; 54:1733-1739

DOI: 10.2967/jnumed.113.120477

Received Jan. 23, 2013; revision accepted May 6, 2013.

For correspondence or reprints contact: Hein J. Verberne, Department of Nuclear Medicine, Academic Medical Center, F2-238, P.O. Box 22700, 1100 DE Amsterdam, The Netherlands.

E-mail: h.j.verberne@amc.uva.n

Published online Aug. 22, 2013.

COPYRIGHT (C) 2013 by the Society of Nuclear Medicine and Molecular Imaging, Inc.
$\mathbf{H}$ (HF) is associated with a prothrombotic state, reflected by an increased risk of arterial and venous thromboembolic events (1-4). The estimated annual incidence of thromboembolic events is $3 \%-5 \%(2,4)$. The increased sympathetic activity present in patients with HF (5) can lead to activation of platelets and increases concentrations of coagulation factors, particularly coagulation factor VIII and von Willebrand factor (VWF) $(1,6)$. Therefore, the increased risk of thromboembolic events in these patients might be, at least in part, related to a hyperactive sympathetic nervous system $(1,4-6)$.

Angiotensin-converting enzyme inhibitors and $\beta$-blockers form the cornerstone of treatment in HF. $\beta$-blockers inhibit the adverse effects of the increased sympathoadrenergic activity, although all $\beta$-blockers may not be equally effective. In a study by Azevedo et al. (7), carvedilol, a nonselective $\beta_{1^{-}}, \beta_{2^{-}}$, and $\alpha_{1^{-}}$-receptor blocker, seemed to be more effective than the selective $\beta_{1}$-receptor blocker metoprolol in reducing sympathetic hyperactivity in patients with HF. However, this study used metoprolol tartrate in a relatively lower target dosage of $100 \mathrm{mg}$ daily, which has been shown to be inferior to carvedilol in the Carvedilol or Metoprolol European Trial (8). In addition, the prothrombotic response on epinephrine or norepinephrine release appears to be $\beta_{2}$-receptor-mediated (6). Nonselective $\beta$-blockers lower the hemostatic response on catecholamine infusions, whereas selective compounds do not (6). Hence, treatment with carvedilol could result in a reduction of the prothrombotic state in HF. Indeed, a large observational study showed that the risk of thromboembolic events was lower in HF patients on nonselective $\beta$-blockers than in those on selective compounds (9).

The efficacy of $\beta$-blockers may be mediated through genetic variation in the $\beta_{2}$-adrenergic receptor. Two highly prevalent haplotypes (Arg16Gln27 and Gly16Glu27) are associated with altered receptor trafficking and downregulation (10). After infusion of isoproterenol, a $\beta$-receptor agonist, venodilatation decreased in persons homozygous for Arg16/Gln27, compared with the Gly16/Glu27 haplotype (11). The other haplotypes acted intermediately. This difference in functionality might explain in part the increased risk for cardiovascular disease $(12,13)$. Patients homozygous for Arg16/ Gln27 who had an acute coronary syndrome had a doubled 3-y mortality rate compared with those homozygous for Gly16/Glu27 
(14). Noteworthy, it was suggested that the $\beta_{2}$-adrenergic receptor genotype also influences the response in left ventricular ejection fraction improvement by carvedilol in HF patients (15).

This led us to the following hypotheses for the present study: sympathetic and thereby prothrombotic activity in patients with $\mathrm{HF}$ is reduced more effectively by the nonselective $\beta$-blocker carvedilol than by the selective $\beta$-blocker metoprolol. Furthermore, this difference in response to $\beta$-blockers is mediated through common $\beta_{2}$-receptor haplotypes.

\section{MATERIALS AND METHODS}

\section{Outcomes}

The primary outcome of this study was cardiac sympathetic activity as measured by ${ }^{123}$ I-meta-iodobenzylguanidine ( $\left.{ }^{123} \mathrm{I}-\mathrm{MIBG}\right)$ myocardial washout. Secondary outcomes regarding sympathetic activity included early and late heart-to-mediastinum ratio in ${ }^{123} \mathrm{I}$-MIBG scintigraphy. For hemostatic activity, we measured markers for coagulation, fibrinolysis, and platelet activation-that is, factor VIII, VWF, fibrinogen, prothrombin fragment $1+2(\mathrm{~F} 1+2)$, d-dimer, and surface presentation of P-selectin on platelets (P-selectin).

\section{Patient Selection}

Patients were recruited from the cardiology outpatient clinics in 6 regional hospitals in The Netherlands. After patients had given written informed consent, they were screened for eligibility, and blood was drawn for DNA collection. Eligible subjects were patients aged 18-80 $\mathrm{y}$, with stable symptoms of chronic HF (New York Heart Association classification I-III) and a left ventricular ejection fraction below $40 \%$ as measured within 6 mo before randomization by nuclear scanning, MR imaging, or cardiac ultrasound. Patients were on stable medical therapy for at least $3 \mathrm{mo}$, including angiotensin-converting enzyme inhibitors or angiotensin receptor blockers (unless contraindicated) and $\beta$-blockers on maximal tolerated dosages. Only patients in sinus rhythm were included in the study. Excluded were patients with an acute coronary event or myocardial revascularization within 3 mo before randomization, severe aortic or mitral valve disease, a requirement for intravenous inotropic therapy, severely uncontrolled hypertension (systolic blood pressure $\geq 170 \mathrm{~mm} \mathrm{Hg}$ or diastolic $\geq 105 \mathrm{~mm} \mathrm{Hg}$ ), known drug or alcohol abuse, a history of poor treatment compliance, or a systemic disease that might complicate management or reduce life expectancy. Pregnant women or women with childbearing potential on inadequate contraception were also excluded. Since many patients with $\mathrm{HF}$ in sinus rhythm used vitamin $\mathrm{K}$ antagonists, we also allowed these patients in the study. Anticoagulant treatment is not expected to affect the primary outcome of sympathetic activity or of platelet activity or levels of VWF, factor VIII, and fibrinogen.

\section{Procedures}

This study had a prospective, randomized, open-label, maskedendpoint design. All eligible patients with HF were screened for their haplotype of the $\beta_{2}$-receptor. Individuals who were homozygous for Gly16/Glu27 and Arg16/Gln27 and met the study inclusion criteria were randomized to carvedilol or metoprolol (Fig. 1), applying block randomization with block sizes of 4 , using the TENALEA randomization service (https://www.tenalea.com). Since included patients were already on $\beta$-blocker treatment, they received an equipotent dosage of carvedilol (Eucardic; Roche) or metoprolol succinate (Selokeen ZOC; AstraZeneca). After the first $\beta$-blocker treatment period of $6 \mathrm{wk}$, patients were switched to the other $\beta$-blocker, again in an equipotent dosage. Equipotent dosages were considered $25 \mathrm{mg}$ twice daily for carvedilol and $200 \mathrm{mg}$ daily for metoprolol succinate (16). To ensure equipotency, the heart rate of patients was monitored $1 \mathrm{wk}$ after they had been switched to the other $\beta$-blocker. If resting heart rate varied by more than 5 beats per minute, the dosage was adjusted accordingly. A comparable heart rate between the $2 \beta$-blockers is important because heart rate reduction is significantly associated with the survival benefit of $\beta$-blockers in HF whereas the dose of $\beta$-blockers may be less important (17). Compliance with $\beta$-blocker treatment was assessed by counting the tablets remaining at the end of the treatment episodes. Sympathetic and hemostatic activity was measured at the end of both treatment periods. As a global estimate of the cardiac condition at the end of each treatment period, N-terminal pro-brain natriuretic peptide (NT-proBNP) concentrations were measured (18). A $\beta$-blocker washout period was not feasible for ethical reasons. The trial complied with the Declaration of Helsinki and was approved by the Ethics Committee of the Academic Medical Center. The present study was the main outcome of a trial registered on www.trialregister.nl (NTR1067).

\section{$\boldsymbol{\beta}$-Receptor Sequencing}

Genomic DNA was isolated from $10 \mathrm{~mL}$ of peripheral blood using an AutopureLS apparatus according to the manufacturer's protocol (Gentra Systems). $\beta_{2}$-adrenergic genotypes at the alleles encoding for positions 16 and 27 were determined by the big-dye terminator sequencing technique, for which a pair of primers was designed (using Primer3; http://frodo.wi.mit.edu/primer3/). Polymerase chain reaction was performed with $50 \mathrm{ng}$ of genomic DNA in a $25-\mu \mathrm{L}$ reaction volume containing $1 \times$ Taq DNA polymerase buffer (Qiagen), a $50 \mu \mathrm{M}$ concentration of each deoxyribonucleoside triphosphate, a $0.4 \mu \mathrm{M}$ concentration of each primer, and 1 unit of Taq DNA polymerase. The thermal cycling conditions were as follows: $95^{\circ} \mathrm{C}$ for $2 \mathrm{~min}$, then 20 cycles of $30 \mathrm{~s}$ at $95^{\circ} \mathrm{C}$ (minus $0.5^{\circ} \mathrm{C}$ during each cycle), $30 \mathrm{~s}$ at $65^{\circ} \mathrm{C}$, and $30 \mathrm{~s}$ at $72^{\circ} \mathrm{C}$ in a polymerase chain reaction apparatus (T3 Biocycler; Biometra). Then, there were 30 cycles of $30 \mathrm{~s}$ at $95^{\circ} \mathrm{C}, 30 \mathrm{~s}$ at $55^{\circ} \mathrm{C}$, and $30 \mathrm{~s}$ at $72^{\circ} \mathrm{C}$. The program ended with $10 \mathrm{~min}$ at $72^{\circ} \mathrm{C}$. The sequence reactions were performed using fluorescently labeled dideoxy chain terminations with a Big Dye Terminator ABI Prism kit (Applied Biosystems) according to the manufacturer's protocol and analyses on an Applied Biosystems automated DNA sequencer (model 3730). Sequences were analyzed with the Sequencher package (GeneCodes Co.).

\section{I-MIBG Scintigraphy}

To block thyroid uptake of free ${ }^{123} \mathrm{I}$, all patients received $100 \mathrm{mg}$ of potassium iodide orally, $1 \mathrm{~h}$ before the injection of ${ }^{123} \mathrm{I}-\mathrm{MIBG}$. After a subsequent resting period of at least $30 \mathrm{~min}$, the patients were in-

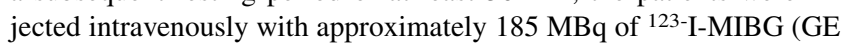
Healthcare). Twenty minutes (early imaging) and $4 \mathrm{~h}$ (delayed imaging) after ${ }^{123} \mathrm{I}-\mathrm{MIBG}$ administration, a 10-min planar anterior image of the chest was acquired. A $20 \%$ energy window was centered on the $159-\mathrm{keV}$ photopeak of ${ }^{123} \mathrm{I}$. Images were acquired using a medium-energy collimator and stored in a $128 \times 128$ matrix. ${ }^{123}$ I-MIBG myocardial

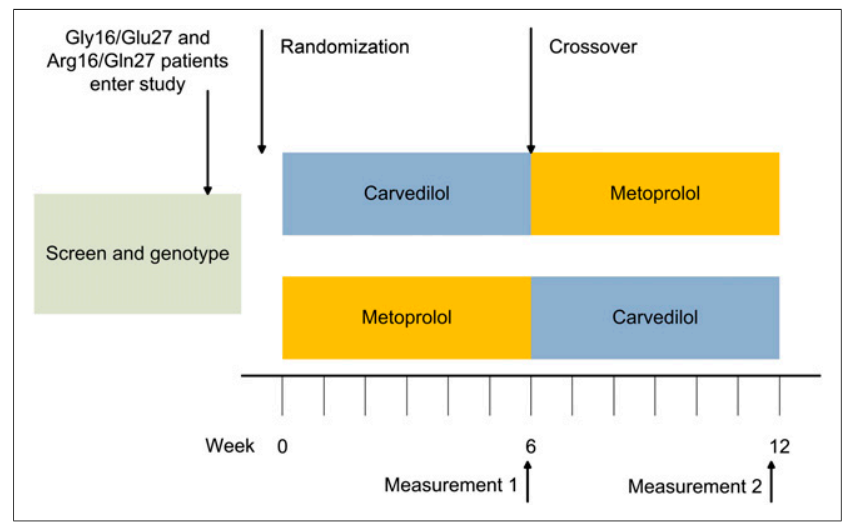

FIGURE 1. Schematic diagram of trial protocol. Measurement = 123/-MIBG scintigraphy and hemostasis. 
activity was measured using a manually drawn region of interest around the left ventricle. Mediastinal activity was measured using a fixed $20 \times$ 20 pixel region of interest placed over the upper mediastinum. Postprocessing was performed on a HERMES workstation (HERMES Medical Solutions). To evaluate ${ }^{123}$ I-MIBG myocardial uptake, the heart $(\mathrm{H})$ to mediastinal $(\mathrm{M})$ ratio was calculated from the early and delayed images. ${ }^{123}$ I-MIBG myocardial washout was defined as the percentage of change in activity from the early and delayed images and is an index of the degree of the sympathetic drive:

$$
\left\{\frac{(\text { early } \mathrm{H} / \mathrm{M}-\text { late } \mathrm{H} / \mathrm{M})}{\text { early } \mathrm{H} / \mathrm{M}}\right\} \times 100 \% \text {. }
$$

This equation implies that increased adrenergic drive is associated with high ${ }^{123}$ I-MIBG myocardial washout.

\section{Measurements of Hemodynamics and Blood Collection}

Between the early and late ${ }^{123}$ I-MIBG scintigraphy, measurements of hemodynamics and hemostasis were performed. To avoid any diurnal and dietary influences, all measurements were performed in the morning after an overnight fast. The subjects were supine, and an intravenous cannula was inserted into the antecubital vein of the right arm and filled with diluted saline. After $10 \mathrm{~min}$ of rest, blood pressure was measured 3 times at 1-min intervals using a validated oscillometric device (Omron 705IT; Omron Healthcare Europe). The first measurement was discarded, and the mean of the last 2 measurements was used for further analysis. Heart rate was monitored by electrocardiography. After $30 \mathrm{~min}$ of rest, blood samples were drawn from the cannula for measurements of hemostasis. The first tube was discarded. Blood for coagulation assays was collected in tubes containing $0.32 \%$ trisodium citrate and processed within $15 \mathrm{~min}$. Plasma was prepared by centrifugation at $2,500 \mathrm{~g}$ twice for $20 \mathrm{~min}$ at $16^{\circ} \mathrm{C}$, followed by storage at $-80^{\circ} \mathrm{C}$ until assays were performed. Blood for NT-proBNP was collected in heparin-containing tubes and prepared by centrifugation at $1,885 \mathrm{~g}$ for $5 \mathrm{~min}$.

NT-proBNP was measured by chemiluminescent immunometric assay (Roche Diagnostics). $\mathrm{F} 1+2$ was measured by enzyme-linked immunosorbent assay (Enzygnost F1 +2 ; Siemens Healthcare Diagnostics); factor VIII activity was determined on an automated coagulation system (BCS-XP; Siemens Healthcare Diagnostics) with reagents and protocols from the manufacturer. The fibrinogen concentration was determined from the change in optical signal during prothrombin time determination on the BCS-XP. D-dimer levels were determined with a particle-enhanced immunoturbidimetric assay (Innovance D-dimer; Siemens Healthcare Diagnostics). VWF antigen levels were tested by enzyme-linked immunosorbent assay with antibodies from DAKO. P-selectin was determined by flow cytometry analysis in whole blood as previously described (19).

\section{Statistical Analysis}

Sample size calculations to detect a clinical relevance difference of $5.0 \%$ in ${ }^{123}$ I-MIBG myocardial washout between the $\beta$-blockers within each haplotype determined that 9 patients with each haplotype were required to provide $90 \%$ power with a 2 -sided $\alpha$ of 0.05 , with the assumption of a $3.0 \% \mathrm{SD}$ (20) and using a paired Student $t$ test.
In primary analyses, differences between the $2 \beta$-blockers were assessed in patients with both haplotypes grouped together. $\beta$-blocker dosages are presented as median and interquartile range. All other outcome data are presented as means \pm SEM. Paired Student $t$ tests were used for within-group comparisons when data were distributed normally. The Wilcoxon rank sum test was used when tests of normality failed. In a sensitivity analysis, the primary outcome of ${ }^{123}$ I-MIBG myocardial washout was assessed in patients without worsening HF symptoms on 1 of the $2 \beta$-blockers. Missing values were imputed with the mean values of the group. Since vitamin K antagonists affect F1 + 2 and d-dimer concentrations, these markers were assessed only in those patients not receiving vitamin $\mathrm{K}$ antagonist treatment. Therefore, F1 + 2 and d-dimer levels were also not assessed within each haplotype, since these subgroups were small. Analyses were performed in SPSS, version 16.0 (IBM).

\section{RESULTS}

\section{Recruitment of Eligible Patients}

Between 2007 and 2009, 86 patients with HF were screened. The $\operatorname{Arg} 16 / \mathrm{Gln} 27$ haplotype was present in 21 patients $(24 \%)$ and Gly16/Glu27 in 13 patients (15\%). Of these 34 patients, 13 were not randomized, for several reasons, mainly because the subjects did not meet the study criteria at the time of randomization (Fig. 2). In the Gly16/Glu27 group, 1 patient withdrew consent 2 wk after randomization because of tiredness after the change in $\beta$-blockade. Hence, 8 patients with the Gly16/Glu27 haplotype and 13 with the Arg16/Gln27 haplotype entered the study protocol (Table 1). Most patients had ischemia-related $\mathrm{HF}(67 \%)$, and $43 \%$ were in New York Heart Association class II. Before randomization, $48 \%$ used metoprolol and $33 \%$ carvedilol. Of the included patients, $57 \%$ were treated with vitamin $\mathrm{K}$ antagonists and $62 \%$ with antiplatelet agents.

During the trial protocol, 5 patients (4 in the $\operatorname{Arg} 16 / G \ln 27$ group and 1 in the Gly16/Glu27 group) experienced an increased shortness of breath after changing from carvedilol to metoprolol,

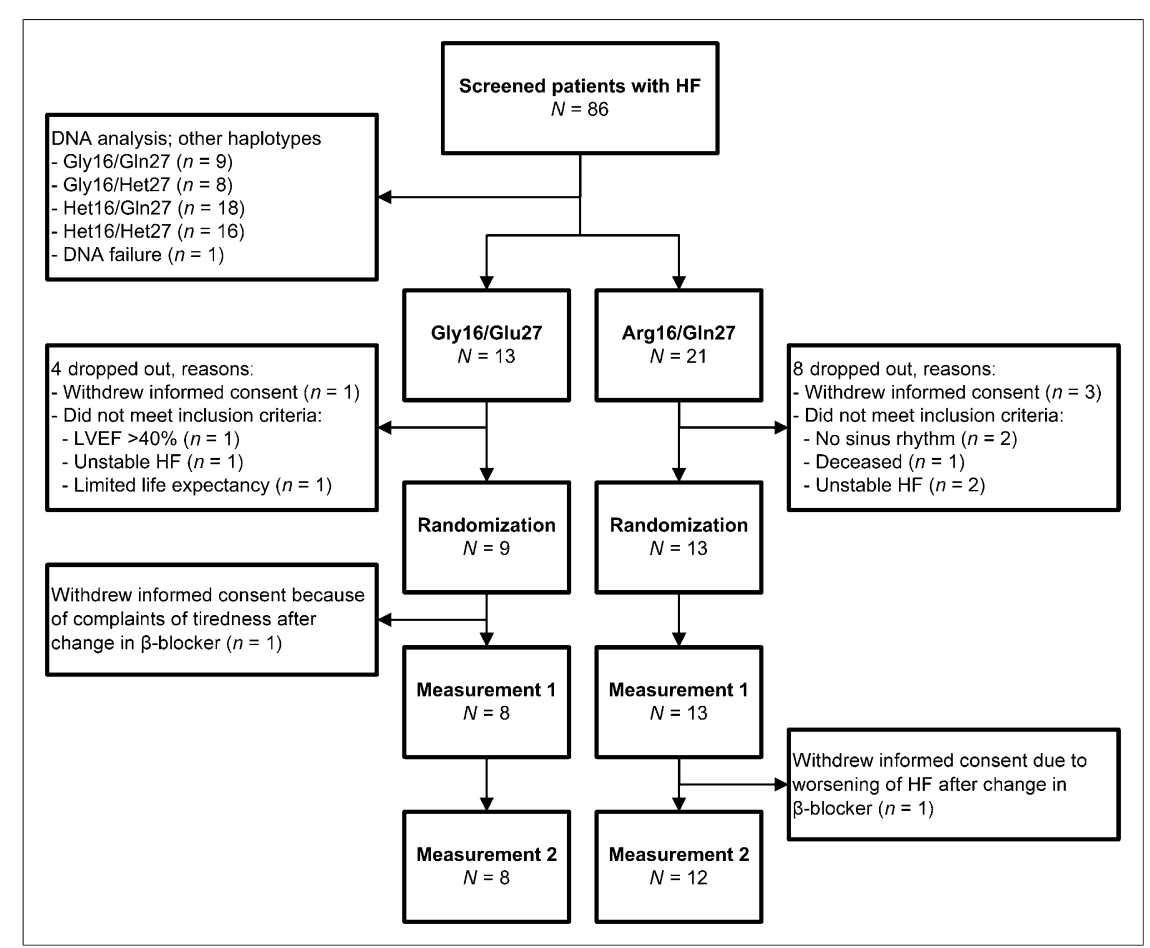

FIGURE 2. Trial profile. LVEF = left ventricular ejection fraction. 
TABLE 1

Baseline Characteristics of Participants by Haplotype

\begin{tabular}{|c|c|c|c|c|}
\hline Characteristic & All patients $(n=21)$ & Gly16/Glu27 ( $n=8)$ & Arg16/GIn27 $(n=13)$ & $P$ \\
\hline Age $(y)$ & $64 \pm 2$ & $70 \pm 3$ & $60 \pm 2$ & 0.02 \\
\hline Male & $13(62)$ & $4(50)$ & 9 (69) & 0.38 \\
\hline Cause of HF & & & & 0.20 \\
\hline Ischemic & $14(67)$ & $4(50)$ & $10(77)$ & \\
\hline \multicolumn{5}{|l|}{ Nonischemic } \\
\hline Hypertensive cardiomyopathy & $2(10)$ & $0(0)$ & $2(15)$ & \\
\hline Dilated cardiomyopathy & $5(24)$ & $4(50)$ & $1(8)$ & \\
\hline LVEF (\%) & $30 \pm 2$ & $28 \pm 4$ & $31 \pm 1$ & 0.47 \\
\hline NYHA classification & & & & 0.87 \\
\hline I & $6(29)$ & $2(25)$ & $4(31)$ & \\
\hline II & $9(43)$ & $4(50)$ & $5(39)$ & \\
\hline III & $6(29)$ & $2(25)$ & $4(31)$ & \\
\hline \multicolumn{5}{|l|}{ Medical history } \\
\hline Hypertension & 7 (33) & $2(25)$ & $5(39)$ & 0.53 \\
\hline Diabetes mellitus & $8(38)$ & $2(25)$ & $6(46)$ & 0.33 \\
\hline Hypercholesterolemia & 7 (33) & $3(38)$ & $4(31)$ & 0.75 \\
\hline$\beta$-blocker before randomization & & & & 0.70 \\
\hline Carvedilol & $7(33)$ & $3(38)$ & $4(31)$ & \\
\hline Metoprolol & $10(48)$ & $4(50)$ & $6(46)$ & \\
\hline Bisoprolol & $2(10)$ & $1(13)$ & $1(8)$ & \\
\hline Nebivolol & $2(10)$ & $0(0)$ & $2(15)$ & \\
\hline \multicolumn{5}{|l|}{ Comedication } \\
\hline RAAS inhibitors & $18(86)$ & $6(75)$ & $12(92)$ & 0.27 \\
\hline Loop diuretic & $17(81)$ & $6(75)$ & $11(85)$ & 0.59 \\
\hline Vitamin $\mathrm{K}$ antagonists & $12(57)$ & $4(50)$ & $8(62)$ & 0.60 \\
\hline Antiplatelet agents & $13(62)$ & $5(63)$ & $8(62)$ & 0.97 \\
\hline Statin & $18(86)$ & $6(75)$ & $12(92)$ & 0.27 \\
\hline Spironolactone & $8(38)$ & $2(25)$ & $6(46)$ & 0.33 \\
\hline Digoxin & $3(14)$ & $2(25)$ & $1(8)$ & 0.27 \\
\hline
\end{tabular}

LVEF = left ventricular ejection fraction; NYHA = New York Heart Association; RAAS = renin-angiotensin-aldosterone-system.

Qualitative data are expressed as numbers, followed by percentages in parentheses; continuous data are expressed as mean \pm SEM.

leading to withdrawal of consent in 1 patient (Arg16/Gln27) $2 \mathrm{wk}$ before the second measurement. In the remaining patients, dosage adjustment of the $\beta$-blocker or a (temporary) increment of loopdiuretic dosage reduced the complaints. Other patients did not report changes in complaints between $\beta$-blockers. The median administered daily dosages were $12.5 \mathrm{mg}$ (interquartile range, 12.537.5) for carvedilol and $75 \mathrm{mg}$ (interquartile range, 50-125) for metoprolol. Compliance with $\beta$-blocker treatment was $99 \%$.

\section{$\beta$-Blocker Treatment and Sympathetic and Hemostatic Activity}

The results of the ${ }^{123}$ I-MIBG scintigraphy are presented in Table 2. Carvedilol significantly reduced ${ }^{123}$ I-MIBG myocardial washout, compared with metoprolol $(12.9 \% \pm 3.9 \%$ vs. $22.1 \% \pm$ $2.8 \%$, respectively, $P=0.003$ ). The early heart-to-mediastinum ratio was lower and the late heart-to-mediastinum ratio was higher during carvedilol treatment, although the difference was nonsignificant.

Table 2 also provides an overview of the hemodynamic measurements and NT-proBNP concentrations. Heart rate was comparable between the $2 \beta$-blockers. When treated with carvedilol, patients had a lower systolic blood pressure than when they were treated with metoprolol (123 $\pm 4 \mathrm{~mm} \mathrm{Hg}$ vs. $129 \pm 3 \mathrm{~mm} \mathrm{Hg}, P=0.01)$. There was no difference in diastolic blood pressure. NT-proBNP levels did not differ significantly.

When the influence of $\beta$-blocker treatment on hemostatic activity was analyzed, carvedilol treatment was associated with lower levels of VWF than was metoprolol treatment $(149 \% \pm 13 \%$ vs. $157 \% \pm 13 \%, P=0.01$, Table 3 ). No differences were observed for factor VIII and fibrinogen concentrations. In patients without vitamin $\mathrm{K}$ antagonists, F1 + 2 levels were comparable between the 2 groups, but d-dimer concentrations tended to be higher during carvedilol treatment $(0.57 \pm 0.25 \mathrm{mg} / \mathrm{L}$ vs. $0.40 \pm 0.17 \mathrm{mg} / \mathrm{L}, P=$ 0.12 ). There was no difference between the $2 \beta$-blockers regarding the marker for platelet activation (P-selectin).

\section{Sensitivity Analysis}

In a sensitivity analysis, we excluded patients with worsening HF on metoprolol treatment $(n=5)$. Also in this analysis, the primary outcome of ${ }^{123}$ I-MIBG myocardial washout was significantly lower in patients when on carvedilol than when on metoprolol $(9.8 \%$ $\pm 4.3 \%$ vs. $18.4 \% \pm 2.7 \%$, respectively, $P=0.01$ ).

\section{$\boldsymbol{\beta}_{\mathbf{2}}$-Receptor Haplotypes}

Homozygous Arg16/Gln27 patients were approximately 10 y younger, more often had an ischemic cause of HF, more often had hypertension and diabetes mellitus, and used more cardiovascular medication, including statins and spironolactone (Table 1).

Compared with metoprolol, carvedilol significantly lowered ${ }^{123} \mathrm{I}-$ MIBG myocardial washout in patients with both haplotypes (Table 4). In Arg16/Gln27 patients, ${ }^{123} \mathrm{I}-\mathrm{MIBG}$ washout was $11.0 \% \pm 5.6 \%$ during carvedilol and $21.8 \% \pm 3.6 \%$ during metoprolol $(P=0.04)$. In Gly16/Glu27 patients, these values were $16.1 \% \pm 4.8 \%$ vs. 
TABLE 2

Carvedilol vs. Metoprolol (6 Weeks of Treatment): ${ }^{123}$ I-MIBG and Hemodynamic Characteristics

\begin{tabular}{|c|c|c|c|}
\hline Characteristic & Carvedilol & Metoprolol & $P$ \\
\hline \multicolumn{4}{|l|}{ 123I-MIBG scintigraphy } \\
\hline 123I-MIBG myocardial washout (\%) & $12.9 \pm 3.9$ & $22.1 \pm 2.8$ & $0.003^{*}$ \\
\hline Early heart-to-mediastinum ratio & $1.73 \pm 0.10$ & $1.85 \pm 0.08$ & 0.05 \\
\hline Late heart-to-mediastinum ratio & $1.50 \pm 0.09$ & $1.43 \pm 0.07$ & 0.24 \\
\hline \multicolumn{4}{|l|}{ Hemodynamic measurements } \\
\hline Systolic BP (mm Hg) & $123 \pm 4$ & $129 \pm 3$ & 0.01 \\
\hline Diastolic BP (mm Hg) & $74 \pm 2$ & $75 \pm 2$ & 0.45 \\
\hline Heart rate (beats/min) & $66 \pm 2$ & $67 \pm 2$ & 0.56 \\
\hline NT-proBNP (ng/L) & $1,032 \pm 297$ & $985 \pm 232$ & 0.69 \\
\hline
\end{tabular}

*Primary outcome of this study.

$\mathrm{BP}=$ blood pressure; IQR = interquartile range.

Data are presented as mean $\pm \operatorname{SEM}(n=21)$, unless otherwise indicated.

$22.6 \% \pm 4.6 \%$, respectively $(P=0.02)$. Also, VWF levels were lower during carvedilol treatment in $\operatorname{Arg} 16 / \mathrm{Gln} 27$ patients $(143 \%$ $\pm 18 \%$ vs. $151 \% \pm 19 \%, P=0.03)$ than in Gly16/Glu27 patients $(159 \% \pm 19 \%$ vs. $166 \% \pm 18 \%, P=0.21)$, although the difference was nonsignificant. Factor VIII, fibrinogen, and P-selectin did not differ between the $2 \beta$-blockers in either haplotype.

\section{DISCUSSION}

In this study, the influence of $6 \mathrm{wk}$ of treatment with carvedilol on cardiac sympathetic and hemostatic activity in stable, chronic HF patients was compared with that of metoprolol succinate, in a crossover design, using equipotent dosages and aiming for comparable heart rates. In addition, we assessed whether the efficacy of $\beta$-blockers was mediated through common $\beta_{2}$-receptor haplotypes. Carvedilol significantly reduced ${ }^{123}$ I-MIBG myocardial washout, compared with metoprolol $(12.9 \% \pm 3.9 \%$ vs. $22.1 \% \pm 2.8 \%$, $P=0.003$ ). This difference in washout was present in both homozygous Arg16/Gln27 and Gly16/Glu27 patients. Finally, during carvedilol treatment, VWF concentrations were lower.

In patients with HF, sympathetic activity is increased to compensate for the loss of cardiac output and circulatory failure. In addition, there is evidence that sympathetic activity induces a procoagulant response (6). Hence, the sympathetic hyperactivity could be an important contributor to the prothrombotic state in $\mathrm{HF}$ $(1,2)$. This study showed that, compared with metoprolol, carvedilol resulted in lower cardiac sympathetic activity and lower VWF levels. This finding is in line with a post hoc analysis of the Car- vedilol or Metoprolol European Trial, in which VWF concentrations showed a decrease during carvedilol treatment, compared with increasing VWF levels in HF patients using metoprolol tartrate (21). Lower levels of VWF are associated with a lower risk of cardiovascular events (22). In combination with the lower cardiac sympathetic activity, this may explain the $16 \%$ lower risk of thromboembolic events in patients with HF when treated with nonselective $\beta$-blockers than when treated with selective $\beta$-blockers (9). In addition, a literature overview suggested a lower thromboembolic event risk in patients with HF when treated with nonselective $\beta$-blockers (23). If these findings are corroborated in a larger, randomized, study, a preference for nonselective $\beta$-blockers may ensue, especially in those HF patients with a high cardiovascular risk.

Carvedilol induced a relatively lower systolic blood pressure than metoprolol. This difference may be explained at least in part by the fact that carvedilol also has $\alpha$-receptor-blocking properties. However, whether this difference in systolic blood pressure is related to a lower risk of thromboembolic events is not known. To our knowledge, there are no direct data available on this subject. On the extreme end of the spectrum, it is known that hypertension is associated with a slightly higher risk of venous thromboembolism (24). However, none of the subjects included in our study had hypertension. Dishy et al. have speculated on this subject and stated that increased sympathetic activity with increased catecholamine concentrations may be associated with hypertension, especially in young persons $(11,25)$. Thus, long-term exposure to elevated concentrations of catecholamines in combination with desensitization to the $\beta_{2}$-adrenergic,

TABLE 3

Comparison of Markers for Hemostasis Between Carvedilol and Metoprolol After 6 Weeks of Treatment

\begin{tabular}{|c|c|c|c|}
\hline Parameter & Carvedilol & Metoprolol & $P$ \\
\hline \multicolumn{4}{|l|}{ Hemostatic parameters in all HF patients } \\
\hline VWF (\%) & $149 \pm 13$ & $157 \pm 13$ & 0.01 \\
\hline Factor VIII (\%) & $155 \pm 9$ & $158 \pm 9$ & 0.46 \\
\hline Fibrinogen (g/L) & $3.7 \pm 0.2$ & $3.8 \pm 0.2$ & 0.22 \\
\hline \multicolumn{4}{|c|}{ Hemostatic parameters in patients without vitamin $\mathrm{K}$ antagonists $(n=9)$} \\
\hline $\mathrm{F} 1+2(\mathrm{pmol} / \mathrm{L})$ & $228 \pm 38$ & $200 \pm 31$ & 0.26 \\
\hline D-dimer (mg/L) & $0.57 \pm 0.25$ & $0.40 \pm 0.17$ & 0.12 \\
\hline Platelet activation status in all patients: P-selectin (\%) & $5.0 \pm 0.8$ & $5.0 \pm 0.6$ & 0.40 \\
\hline
\end{tabular}

Data are presented as mean $\pm \operatorname{SEM}(n=21)$ 
TABLE 4

123/-MIBG Myocardial Washout and Hemostatic Outcomes by $\beta$-Blocker Treatment and Haplotype

\begin{tabular}{ccc}
\hline \multicolumn{1}{c}{ Parameter } & Carvedilol & Metoprolol \\
\hline 123I-MIBG myocardial washout (\%) & & \\
Arg16/GIn27 $(n=13)$ & $11.0 \pm 5.6$ & $21.8 \pm 3.6^{*}$ \\
Gly16/Glu27 $(n=8)$ & $16.1 \pm 4.8$ & $22.6 \pm 4.6^{*}$ \\
Hemostasis & & \\
VWF (\%) & $143 \pm 18$ & $151 \pm 19^{*}$ \\
Arg16/Gln27 $(n=13)$ & $159 \pm 19$ & $166 \pm 18$ \\
Gly16/Glu27 $(n=8)$ & & \\
Factor VIII (\%) & $147 \pm 11$ & $147 \pm 10$ \\
Arg16/Gln27 $(n=13)$ & $167 \pm 17$ & $175 \pm 15$ \\
Gly16/Glu27 $(n=8)$ & $3.5 \pm 0.2$ & $3.5 \pm 0.2$ \\
Fibrinogen (g/L) & $4.1 \pm 0.4$ & $4.2 \pm 1.0$ \\
Arg16/Gln27 $(n=13)$ & & \\
Gly16/Glu27 $(n=8)$ & $5.0 \pm 1.3$ & $5.1 \pm 0.8$ \\
P-selectin $(\%)$ & $4.9 \pm 0.6$ & $4.8 \pm 0.7$ \\
Arg16/Gln27 $(n=13)$ &
\end{tabular}

${ }^{\star} P<0.05$ for carvedilol versus metoprolol.

Data are presented as mean \pm SEM.

vasodilative effects of epinephrine may increase blood pressure, because under these conditions the $\alpha$-adrenergic pressor effect of epinephrine and norepinephrine would be unopposed by vasodilatation. Increased sympathetic activity and enhanced vasoconstriction have also been implicated in the pathogenesis of preeclampsia (26). Dishy et al. went even further and stated that if the polymorphisms they studied have "similar effects on desensitization in other tissues, including the heart, they may influence the clinical course and survival of patients with congestive heart failure" (11). However, it remains to be established whether the relative small difference in systolic blood pressure between carvedilol and metoprolol may lead to a lower risk of thromboembolic events.

The lower VWF concentrations during carvedilol treatment may be the result of the lower sympathetic activity brought about by carvedilol, which could lead to a reduced prothrombotic state. Factor VIII levels, which are more consistently associated with epinephrine and norepinephrine (6), were, however, unaffected by carvedilol. Carvedilol also acts as a free-radical scavenger, has vasodilator properties due to its $\alpha_{1}$-receptor blocking capacities, and has antiproliferative properties, all of which may improve endothelial function and thus lower VWF concentrations (27).

We measured cardiac sympathetic activity using ${ }^{123}$ I-MIBG scintigraphy, a method that is becoming increasingly generally accepted (28). Patients with HF and an increased ${ }^{123}$ I-MIBG myocardial washout tend to have a poorer prognosis (29). Previous studies found a reduced cardiac norepinephrine spillover by nonselective $\beta$-blockers such as carvedilol and propranolol (a nonselective $\beta_{1}$ - and $\beta_{2}$-receptor blocker) but not by metoprolol tartrate $(7,30)$. In addition, the fact that Azevedo et al. (7) observed no difference in muscle sympathetic nerve activity during carvedilol therapy suggests a $\beta_{2}$-receptorenhancing norepinephrine release in the presynaptic cleft. Our study extended these observations by showing a lower ${ }^{123}$ I-MIBG washout during carvedilol treatment but was the first to compare carvedilol with the currently advised treatment of metoprolol succinate (31).

Finally, we assessed whether sympathetic and hemostatic activity in HF patients is influenced by functional, common $\beta_{2}$-receptor haplotypes. We carefully selected patients who were homozygous for $\operatorname{Arg} 16 / G \ln 27$ or Gly16/Glu27. Noteworthy, Arg16/Gln27 patients were on average $10 \mathrm{y}$ younger, more often had an ischemic cause of their cardiomyopathy, and more often had hypertension and diabetes mellitus. Apart from the difference in age, these observations are in line with previous studies $(12,32,33)$. The reason for the difference in age is unclear, since the $\operatorname{Arg} 16 / \mathrm{G} \ln 27$ haplotype does not influence survival in patients with HF (34) and seems not to be a risk factor for HF (35). A previous retrospective study suggested an improved left ventricular ejection fraction in Gly16/Glu27 individuals when treated with carvedilol (15), but this trial was contradicted by another study (36). Given the differences in baseline in our study, it is difficult to directly compare patients with the 2 haplotypes. Nevertheless, the effect of carvedilol on sympathetic activity was clearly present in both haplotypes. One caveat of the receptor haplotype data is that our study was not designed to compare baseline imaging off medical therapy, for obvious ethical reasons. It may be that there are differential baseline MIBG ratios for the 2 haplotypes and that it is the change from baseline conferred by the $\beta$-blockers that reveals the relative differences in haplotype response. More study is needed with respect to understanding the haplotype contributions to pharmacotherapy and signaling.

An important strength of this study was the crossover design for the comparison between $\beta$-blockers, which reduces the influence of confounding covariates and limits the number of patients to have sufficient power. Second, by randomizing patients, the possibility of "order" and "carry-over" effects were likely to be equally distributed between the $2 \beta$-blockers.

There were also some limitations that need to be discussed. This was the first study that prospectively assessed differences between $2 \beta$-blockers on hemostatic parameters. We observed significantly lower concentrations of VWF during carvedilol treatment, but other markers were not clearly different. However, this study was not powered to detect significant differences in hemostatic parameters (e.g., d-dimer), and future studies should assess more robustly whether differences in these markers between the 2 $\beta$-blockers are important for clinical decisions. A second limitation was that we did not include patients with other variants of the $\beta_{2}$-receptor. However, other variants are likely to act intermediately $(11,14)$. Third, for practical reasons, treatment was openlabel. To limit the possibility of placebo effects, patients were unaware of which $\beta$-blocker was assumed to be more effective, and all investigators were masked to treatment. Fourth, although this study assessed haplotypes of the $\beta_{2}$-receptor, these still may be not the causal single-nucleotide polymorphisms but in high linkage disequilibrium with them. Finally, administered dosages of the $\beta$-blockers could be considered low, since target doses are $25 \mathrm{mg}$ twice daily for carvedilol and $200 \mathrm{mg}$ daily for metoprolol. However, the dosages in our study were comparable to those observed in the EuroHeart Failure Survey and thus represent daily clinical practice (37).

Based on the assumption that $200 \mathrm{mg}$ of metoprolol equals 25 $\mathrm{mg}$ of carvedilol, the average dose given to our subjects did not exactly follow that ratio (factor of 8). Therefore, we might have given a relative underdose of metoprolol or, vice versa, a relative overdose of carvedilol. However, the resting heart rate did not differ between carvedilol and metoprolol, but systolic blood pressure did (123 $\pm 4 \mathrm{~mm} \mathrm{Hg}$ vs. $129 \pm 3 \mathrm{~mm} \mathrm{Hg}$, carvedilol vs. metoprolol, respectively, $P=0.01$ ). This difference in systolic blood pressure is probably related to the fact that carvedilol is not a pure $\beta$ blocker but also has $\alpha$-receptor blocking properties. 


\section{CONCLUSION}

In patients with chronic, stable HF, carvedilol significantly reduced cardiac sympathetic activity as measured by ${ }^{123}$ I-MIBG scintigraphy after 6 wk of treatment, when compared with metoprolol. In addition, we observed lower VWF levels during carvedilol treatment. These findings could explain the lower risk of thromboembolic events as shown with nonselective $\beta$-blocker treatment in HF (9). However, a causal relationship between sympathetic activity and the lower VWF remains unclear. The observed differences between $\beta$-blockers were present in both Arg16/Gln27 and Gly16/Glu27 patients, suggesting that these haplotypes are less important in $\beta$ blocker-treated, stable HF patients. To establish whether treatment with a nonselective $\beta$-blocker such as carvedilol indeed reduces the prothrombotic state in HF and thus the risk of thromboembolic events, a larger prospective study is needed.

\section{DISCLOSURE}

The costs of publication of this article were defrayed in part by the payment of page charges. Therefore, and solely to indicate this fact, this article is hereby marked "advertisement" in accordance with 18 USC section 1734 . This study was supported by an unrestricted grant from the Netherlands Heart Foundation (NHF-2006B180). No other potential conflict of interest relevant to this article was reported.

\section{ACKNOWLEDGMENTS}

We thank Petra van Donk, Tonny Veelenturf, and Ronald Zwart for their assistance in guiding the $\beta$-blocker treatment; Annemarie van der Geer for her assistance in database management; Jorge Peter for his assistance in sequencing the $\beta_{2}$-receptor gene; Lizzy Brewster for epidemiologic and statistical advice; Harry Büller for critical revision of the manuscript; and Astrid van Moerkerken for processing the ${ }^{123} \mathrm{I}-$ MIBG scintigrams. We thank Jan Tonnis Keijer, cardiologist, TerGooi Hospitals; Hans Verheul, cardiologist, Flevoziekenhuis; and Andre de Groot, cardiologist, Slotervaart hospital, for their assistance in this study.

\section{REFERENCES}

1. Chung I, Lip GY. Platelets and heart failure. Eur Heart J. 2006;27:2623-2631.

2. de Peuter OR, Kok WE, Torp-Pedersen C, Buller HR, Kamphuisen PW. Systolic heart failure: a prothrombotic state. Semin Thromb Hemost. 2009;35:497-504.

3. Gibbs CR, Blann AD, Watson RD, Lip GY. Abnormalities of hemorheological, endothelial, and platelet function in patients with chronic heart failure in sinus rhythm: effects of angiotensin-converting enzyme inhibitor and beta-blocker therapy. Circulation. 2001;103:1746-1751.

4. Lip GY, Gibbs CR. Does heart failure confer a hypercoagulable state? Virchow's triad revisited. J Am Coll Cardiol. 1999;33:1424-1426.

5. Cohn JN, Levine TB, Olivari MT, et al. Plasma norepinephrine as a guide to prognosis in patients with chronic congestive heart failure. N Engl J Med. 1984;311:819-823.

6. Von Känel R, Dimsdale JE. Effects of sympathetic activation by adrenergic infusions on hemostasis in vivo. Eur J Haematol. 2000;65:357-369.

7. Azevedo ER, Kubo T, Mak S, et al. Nonselective versus selective beta-adrenergic receptor blockade in congestive heart failure: differential effects on sympathetic activity. Circulation. 2001;104:2194-2199.

8. Poole-Wilson PA, Swedberg K, Cleland JG, et al. Comparison of carvedilol and metoprolol on clinical outcomes in patients with chronic heart failure in the Carvedilol Or Metoprolol European Trial (COMET): randomised controlled trial. Lancet. 2003;362:7-13.

9. de Peuter OR, Souverein PC, Klungel OH, Buller HR, de Boer A, Kamphuisen PW. Non-selective vs. selective beta-blocker treatment and the risk of thrombo-embolic events in patients with heart failure. Eur J Heart Fail. 2011;13:220-226.

10. Liggett SB. beta ${ }_{2}$-adrenergic receptor pharmacogenetics. Am J Respir Crit Care Med. 2000;161(suppl):S197-S201.

11. Dishy V, Sofowora GG, Xie HG, et al. The effect of common polymorphisms of the beta2-adrenergic receptor on agonist-mediated vascular desensitization. N Engl J Med. 2001;345:1030-1035.
12. Castellano M, Rossi F, Giacche M, et al. Beta 2 -adrenergic receptor gene polymorphism, age, and cardiovascular phenotypes. Hypertension. 2003;41:361-367.

13. Heckbert SR, Hindorff LA, Edwards KL, et al. Beta2-adrenergic receptor polymorphisms and risk of incident cardiovascular events in the elderly. Circulation. 2003;107:2021-2024.

14. Lanfear DE, Jones PG, Marsh S, Cresci S, McLeod HL, Spertus JA. Beta2adrenergic receptor genotype and survival among patients receiving beta-blocker therapy after an acute coronary syndrome. JAMA. 2005;294:1526-1533.

15. Kaye DM, Smirk B, Williams C, Jennings G, Esler M, Holst D. Beta-adrenoceptor genotype influences the response to carvedilol in patients with congestive heart failure. Pharmacogenetics. 2003;13:379-382.

16. Swedberg K, Cleland J, Dargie H, et al. Guidelines for the diagnosis and treatment of chronic heart failure: executive summary (update 2005)—The Task Force for the Diagnosis and Treatment of Chronic Heart Failure of the European Society of Cardiology. Eur Heart J. 2005;26:1115-1140.

17. McAlister FA, Wiebe N, Ezekowitz JA, Leung AA, Armstrong PW. Meta-analysis: beta-blocker dose, heart rate reduction, and death in patients with heart failure. Ann Intern Med. 2009;150:784-794.

18. Richards AM, Crozier IG, Yandle TG, Espiner EA, Ikram H, Nicholls MG. Brain natriuretic factor: regional plasma concentrations and correlations with haemodynamic state in cardiac disease. Br Heart J. 1993;69:414-417.

19. Reuwer AQ, Nieuwland R, Fernandez I, et al. Prolactin does not affect human platelet aggregation or secretion. Thromb Haemost. 2009;101:1119-1127.

20. Somsen GA, Verberne HJ, Fleury E, Righetti A. Normal values and within-subject variability of cardiac I-123 MIBG scintigraphy in healthy individuals: implications for clinical studies. J Nucl Cardiol. 2004;11:126-133.

21. Boman K, Jansson JH, Nilsson T, Swedberg K, Cleland JG, Poole-Wilson P. Effects of carvedilol or metoprolol on PAI-1, tPA-mass concentration or Von Willebrand factor in chronic heart failure: a COMET substudy. Thromb Res. 2010;125:e46-e50.

22. Hamsten A. The hemostatic system and coronary heart disease. Thromb Res. 1993;70:1-38.

23. de Peuter OR, Lussana F, Peters RJ, Buller HR, Kamphuisen PW. A systematic review of selective and non-selective beta blockers for prevention of vascular events in patients with acute coronary syndrome or heart failure. Neth J Med. 2009;67:284-294.

24. Ageno W, Becattini C, Brighton T, Selby R, Kamphuisen PW. Cardiovascular risk factors and venous thromboembolism: a meta-analysis. Circulation. 2008;117:93-102.

25. Goldstein DS. Plasma catecholamines and essential hypertension: an analytical review. Hypertension. 1983;5:86-99.

26. Schobel HP, Fischer T, Heuszer K, Geiger H, Schmieder RE. Preeclampsia: a state of sympathetic overactivity. N Engl J Med. 1996;335:1480-1485.

27. Feuerstein GZ, Ruffolo RR Jr. Carvedilol, a novel multiple action antihypertensive agent with antioxidant activity and the potential for myocardial and vascular protection. Eur Heart J. 1995;16(suppl F):38-42.

28. Jacobson AF, Senior R, Cerqueira MD, et al. Myocardial iodine-123 metaiodobenzylguanidine imaging and cardiac events in heart failure: results of the prospective ADMIRE-HF (AdreView Myocardial Imaging for Risk Evaluation in Heart Failure) study. J Am Coll Cardiol. 2010;55:2212-2221.

29. Verberne HJ, Brewster LM, Somsen GA, van Eck-Smit BL. Prognostic value of myocardial ${ }^{123}$ I-metaiodobenzylguanidine (MIBG) parameters in patients with heart failure: a systematic review. Eur Heart J. 2008;29:1147-1159.

30. Newton GE, Parker JD. Acute effects of beta 1-selective and nonselective betaadrenergic receptor blockade on cardiac sympathetic activity in congestive heart failure. Circulation. 1996;94:353-358.

31. Hunt SA. ACC/AHA 2005 guideline update for the diagnosis and management of chronic heart failure in the adult: a report of the American College of Cardiology/American Heart Association Task Force on Practice Guidelines (Writing Committee to Update the 2001 Guidelines for the Evaluation and Management of Heart Failure). J Am Coll Cardiol. 2005;46:e1-e82.

32. Chang TJ, Tsai MH, Jiang YD, et al. The Arg16Gly polymorphism of human beta2-adrenoreceptor is associated with type 2 diabetes in Taiwanese people. Clin Endocrinol (Oxf). 2002;57:685-690.

33. Gjesing AP, Andersen G, Burgdorf KS, et al. Studies of the associations between functional beta2-adrenergic receptor variants and obesity, hypertension and type 2 diabetes in 7,808 white subjects. Diabetologia. 2007;50:563-568.

34. Sehnert AJ, Daniels SE, Elashoff M, et al. Lack of association between adrenergic receptor genotypes and survival in heart failure patients treated with carvedilol or metoprolol. J Am Coll Cardiol. 2008;52:644-651.

35. Covolo L, Gelatti U, Metra M, et al. Role of beta1- and beta2-adrenoceptor polymorphisms in heart failure: a case-control study. Eur Heart J. 2004;25:1534-1541.

36. de Groote P, Helbecque N, Lamblin N, et al. Association between beta-1 and beta- 2 adrenergic receptor gene polymorphisms and the response to beta-blockade in patients with stable congestive heart failure. Pharmacogenet Genomics. 2005;15:137-142.

37. Komajda M, Follath F, Swedberg K, et al. The EuroHeart Failure Survey programme: a survey on the quality of care among patients with heart failure in Europe. Part 2: treatment. Eur Heart J. 2003;24:464-474. 